\title{
Comparison of Sono-guided Capsular Distension with Fluoroscopically Capsular Distension in Adhesive Capsulitis of Shoulder
}

\author{
Ki Deok Park, M.D., Hee Seung Nam, M.D. ${ }^{1}$, Tai Kon Kim, M.D. ${ }^{2}$, Seong Hoon Kang, M.D. ${ }^{3}$, \\ Min Ho Lim, M.D. ${ }^{3}$, Yongbum Park, M.D. ${ }^{3}$
}

\begin{abstract}
Department of Rehabilitation Medicine, Gachon University of Medicine and Science, Gil Medical Center, Incheon 405-760, ${ }^{1}$ Hallym University College of Medicine, Seoul 134-701, ${ }^{2}$ Hanyang University College of Medicine, Seoul 133-791,

${ }^{3}$ Sanggye Paik Hospital, Inje University College of Medicine, Seoul 139-707, Korea
\end{abstract}

Objective To investigate the short-term effects and advantages of sono-guided capsular distension, compared with fluoroscopically guided capsular distension in adhesive capsulitis of shoulder.

Method In this prospective, randomized, and controlled trial, 23 patients (group A) were given an intra-articular injection of a mixture of $0.5 \%$ lidocaine $(9 \mathrm{ml})$, contrast dye $(10 \mathrm{ml})$, and triamcinolone $(20 \mathrm{mg})$; they received the injection once every 2 weeks, for a total of 6 weeks, under sono-guidance. Twenty-five patients (group B) were treated similarly, under fluoroscopic guidance. Instructions for the self-exercise program were given to all subjects, without physiotherapy and medication. Effects were then assessed using a visual numeric scale (VNS), and the shoulder pain and disability index (SPADI), as well as a range of shoulder motion examinations which took place at the beginning of the study and 2 and 6 weeks after the last injection. Incremental cost-effective ratio (ICER), effectiveness, preference, and procedure duration were evaluated 6 weeks post-injection.

Results The VNS, SPADI, and shoulder motion range improved 2 weeks after the last injection and continued to improve until 6 weeks, in both groups. However, no statistical differences in changes of VNS, SPADI, ROM, and effectiveness were found between these groups. Patients preferred sono-guided capsular distension to fluoroscopically guided capsular distension due to differences in radiation hazards and positional convenience. Procedure time was shorter for sono-guided capsular distension than for fluoroscopically guided capsular distension.

Conclusion Sono-guided capsular distension has comparable effects with fluoroscopically guided capsular distension for treatment of adhesive capsulitis of the shoulder. Sono-guided capsular distension can be substituted for fluoroscopic capsular distension and can be advantageous from the viewpoint of radiation hazard mitigation, time, cost-effectiveness and convenience.

Key Words Sono-guided, Fluoroscopically, Capsular distension, Adhesive capsulitis

Received January 20, 2011; Accepted October 17, 2011

Corresponding author: Yongbum Park

Department of Rehabilitation Medicine, Sanggye Paik Hospital, Inje University College of Medicine, 761-1, Sanggye 6,7 dong, Nowon-gu, Seoul 139707, Korea

Tel: +82-2-950-1390, Fax: +82-2-935-3076, E-mail: swc328@naver.com

(a) This is an open-access article distributed under the terms of the Creative Commons Attribution Non-Commercial License (http://creativecommons. org/licenses/by-nc/3.0) which permits unrestricted noncommercial use, distribution, and reproduction in any medium, provided the original work is properly cited.

Copyright $\odot 2012$ by Korean Academy of Rehabilitation Medicine 


\section{INTRODUCTION}

Adhesive capsulitis is described as synovial hypertrophy and contraction, along with chronic inflammation of synovium with a relatively small amount of synovial fluid around the humeral head. More than $2 \%$ of the general population is known to have the disease; women over 50 years of age are more likely to present with this condition. ${ }^{1,2}$ Currently, several methods of treatment, such as physical therapy, pharmaceutical intervention, intraarticular injections, and exercise therapy are in clinical use, although the different methods show no differences in efficacy. ${ }^{3-5}$ The capsular distension method has a therapeutic effect, via rupture of the stiffened capsule, by hydropressure. ${ }^{6}$

Under fluoroscopic guidance with contrast media injection, capsular distension was performed. After confirming the spinal needle's intra-articular placement in the articular cavity, contrast-mixed saline was injected until the pressure was sufficiently reduced to rupture the capsule. The rupture can be confirmed by the leakage of contrast media outside the capsule, and thisrupture usually takes place below the supscapular muscles and in the biceps' tendon sheath. The success rate of the procedure is reported at $52-82 \% .^{5-8}$

Recently, ultra-sonography is more widely used in the diagnosis and treatment of musculoskeletal disease. The injection method, using a blind anatomical marker, when previously performed, had the limitation of delivering the drug into precisely the expected space. This method also leads to limitations in evaluating treatment efficacy. The $\mathrm{X}$-ray guided injection method has the advantage of delivering drugs to the targeted space but the disadvantage of radiological exposure for both patients and clinicians. Additionally, this method can only be performed in a well-equipped healthcare service. On the other hand, ultra-sonography can be easily used in an outpatient setting, without the fear of radiological exposure.

Gam et al. ${ }^{5}$ compared the efficacy of the capsular distension method with the glucocorticoid injection to glucocorticoid alone. Their results showed a definite improvement in range of motion along with the actual capsular distension seen in US. ${ }^{5}$ However, few studies have been conducted to investigate the pros and cons of sonoguided capsular distension, compared with fluoroscopically guided capsular distension in patients. This study aims to evaluate this very issue.

\section{MATERIALS AND METHODS}

\section{Study subjects}

Patients visiting our rehabilitation outpatient department complainings of shoulder pain and limitation in motion, from January 2009 to June 2010, were initially selected. They were evaluated using a medical interview, physical exam, radiological exam, and musculoskeletal ultrasonography (US) in order to diagnose adhesive capsulitis. Among the patients diagnosed with adhesive capsulitis, 48 with remaining symptoms, after a 3 monthtreatment period, were enrolled in the study. All patients were randomly assigned into two groups: group A for sono-guided capsular distension and group B for fluoroscopically guided capsular distension. Patients meeting study inclusion criteria ${ }^{9-11}$ were those with 1 or 2 grade adhesive capsulitis, with symptom durations of 3 months to 2 years, or those with difficulties in two or more of the following: flexion, abduction, and/or external rotation presenting limitation of more than 30 degrees in passive and active ranges of motion, compared to the normal side with regard to range of motion. Exclusion criteria ${ }^{9-11}$ encompassed the following: complete rotator cuff tear as seen with US, past traumatic shoulder injury requiring hospitalization in the prior 6 months, severe mental illness, age of less than 18 years or more than 70 , degenerative arthritis in the shoulder (detected by radiological studies), systemic inflammatory disease, current use of anti-coagulant medications, diabetes mellitus, having known side effects with lidocaine or contrast media, and current infectious disease or suspected infection.

\section{Method}

Randomization and injection frequency: After the acquisition of patients' basic characteristics, participants went through one of two treatment modalities. The participants were assigned into two groups with the random block assignment method; patients were lined up to be assigned. Four patient-blocks were further subdivided into groups $A$ and $B$, with the use of a 6 serial method: 'AABB, ABAB, BAAB, BABA, BBAA, ABBA'. For every fourth participant, one method among 6 was randomly selected and assigned to that individual. Group A, under US guidance, was treated with $20 \mathrm{cc}$ of the injection drug, 
consisting of $10 \mathrm{cc}$ non-ionic contrast media Omnipaque 300; GE Healthcare (Carrigtwohill Co., Cork, Ireland) mixed with $0.5 \%$ lidocaine $10 \mathrm{cc}$ containing $20 \mathrm{mg}$ triamcinolone. Group B received fluoroscopically guided capsular distension with the same injection material. All patients received the procedure 3 times with 2 week intervals between treatments. Group A had additional radiological confirmation for proper intra-articular injection and evidence of capsular rupture.

Injection method: In sono-guided capsular distension, the injection was performed using a posterolateral approach with Accuvix $\mathrm{XQ}^{\circledR}$ (Medison, Seoul, Korea) and a 6-12 MHz probe, with patients sitting up during treatment. Proper intra-articular injection was evaluated, with evidence of capsular distension by monitoring during the procedure followed by radiological exam (Fig. 1).

In fluoroscopically guided capsular distension, the humeral head was captured with a C-arm (KOMED, Kwangju, Korea) adjusted in a cephalic-to-caudal angle, with patients in a prone position with a pillow underneath the affected shoulder. After aseptic preparation, a 22 gauge, 3.5 inch spinal needle Spinocan ${ }^{\circledR}$ (BRAUN, Melsungen, Germany) was placed superior to the anatomical neck of the humerus, and a solution containing non-ionic contrast media (Omnipaq) was injected under simultaneous fluoroscopic imaging (Fig. 2).

All injection methods were performed by the same clinician, and no further physical therapy or medication was added. Only shoulder self-exercise prior to the procedures was permitted. Additionally, acetaminophen, with no anti-inflammatory effect, or ice massages were permitted if patients complained of pain at the injection site. Acetaminophen use during the follow-up period was also allowed.

Evaluation of treatment efficacy: The evaluation was completed a total of three times, before the first injection followed by tests, and at the second and sixth weeks after completion of procedures. The degree of shoulder pain was graded with a visual numeric scale (VNS) with 1-cm increments between 0-10 cm. Functional disability was assessed by the 'Shoulder Pain and Disability Index' (SPADI) proposed by Roach et al. ${ }^{12}$ with scoring from 0 to 80 , for which 0 represents absence of disability and point 10 represents severe disability that is in need of help for moving (in each of 8 categories). Range of motion in flexion, abduction, and external rotation was assessed both for passive and active movements. Flexion and abduction were examined in a supine position, with the main joint extended from the immobilized trunk using a 180-degree system. External rotation was examined with the shoul-
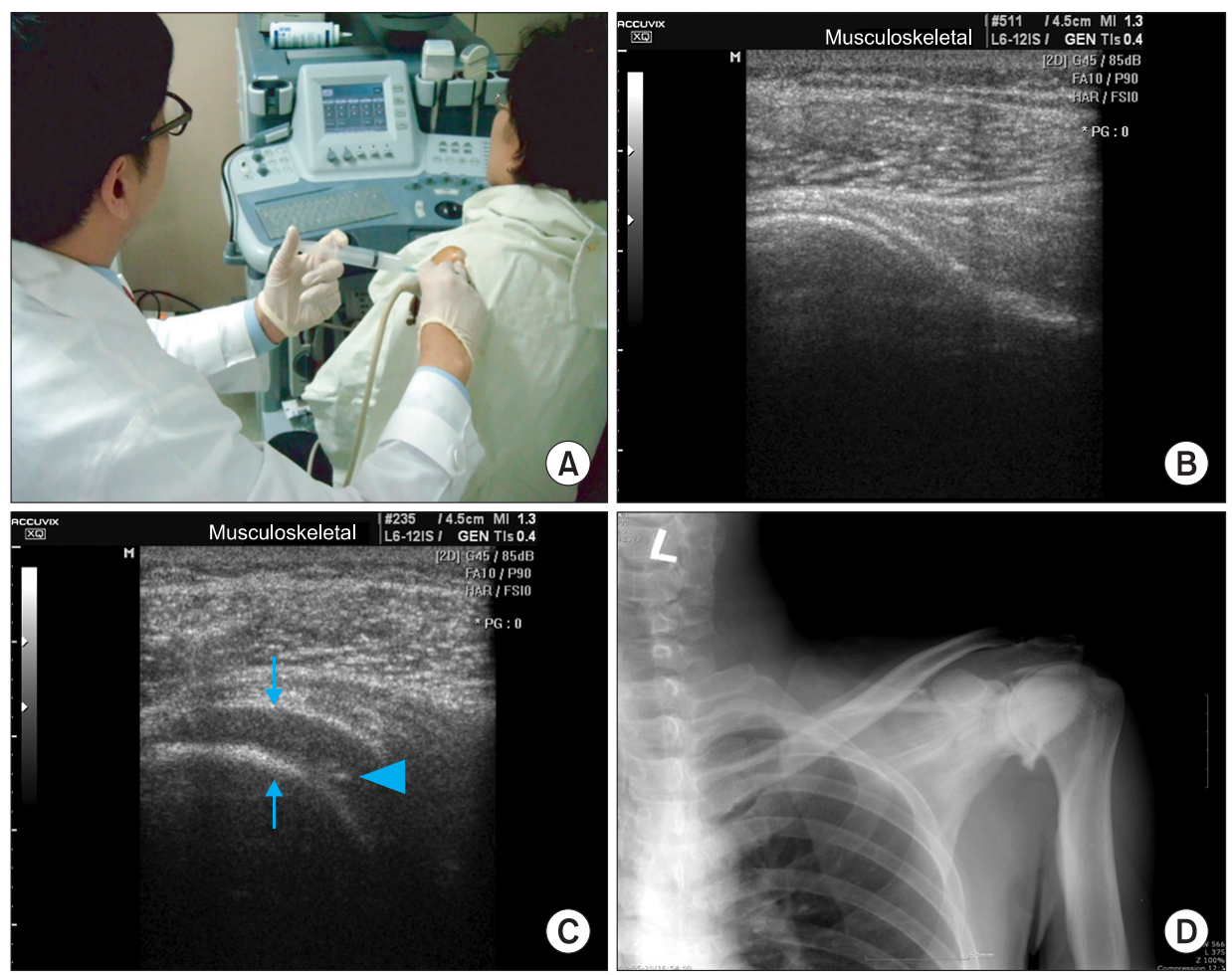

Fig. 1. Sono-guided capsular distension. (A) Sono-guided capsular distension by the posterior lateral approach. (B) Before Sono-guided intra-articular injection at glenohumeral joint. (C) After sonoguided injection with capsular distension (arrow). The needle tip (arrowhead). (D) Shoulder posterioranterior view. After steroid injection with capsular distension. 

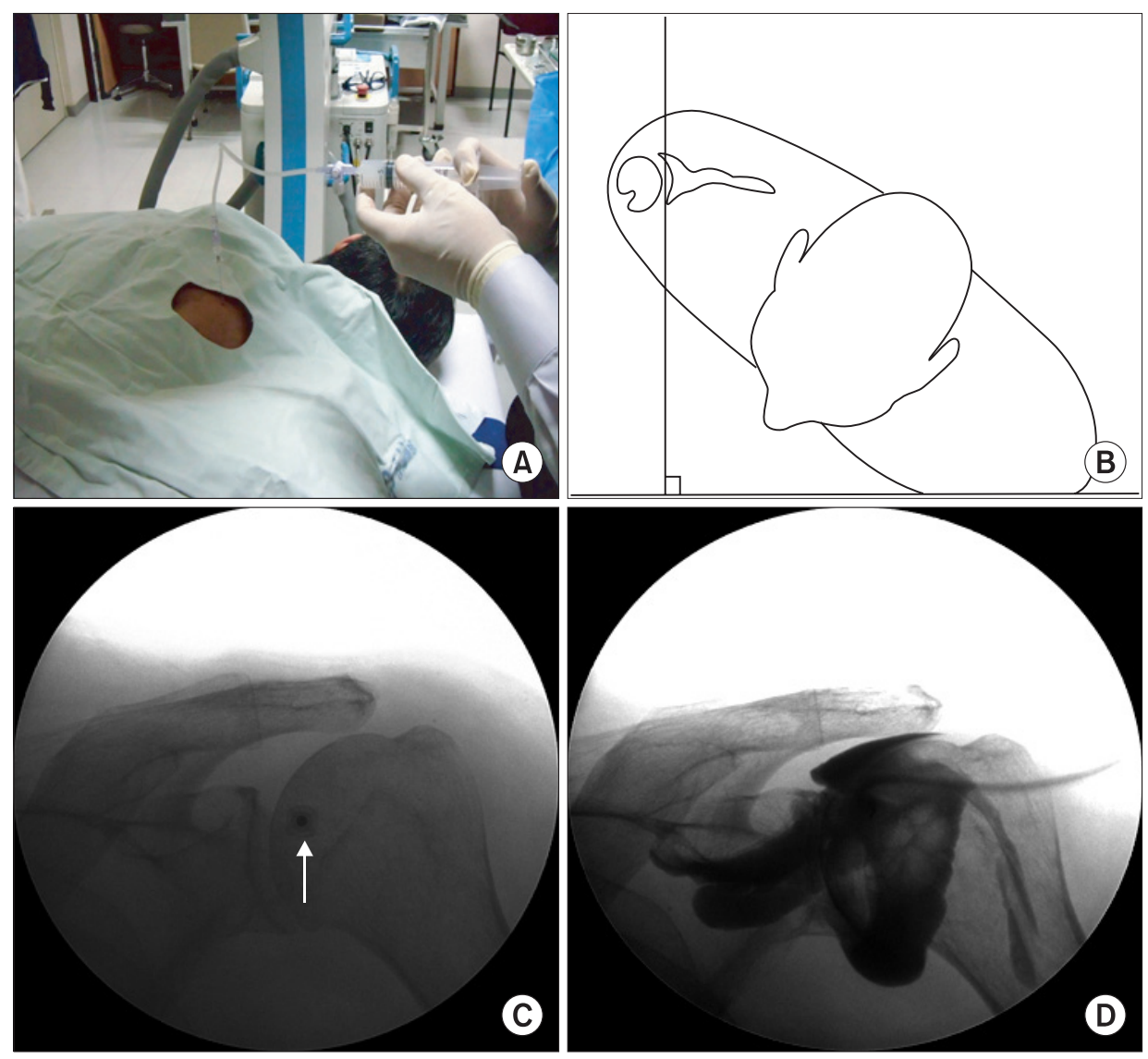

Fig. 2. Fluoroscopically guided capsular distension. (A) Fluoroscopically guided capsular distension by the posterior approach. (B) Drawing shows patient in prone position for posterior approach. (C) Shoulder posterior-anterior view. The needle tip (arrow) was inserted above anatomical neck of humerus (D) Shoulder posterior-anterior view. After steroid injection with capsular distension. der joint abducted 90 degrees and the main joint flexed 90 degrees, as measured with a goniometer.

Comparison in patient satisfaction, efficacy, time consumed, cost-effectiveness, and side effects: Data using patient questionnaires were collected by a doctor who had no knowledge of this study. Patients' satisfaction levels after the completion of 3 injections during a 6 week period were expressed as "excellent," "good," "fair," and "poor". Patients' preference levels for the procedure were expressed in their answers to the question, "Would you choose to receive the same procedure again after being informed of side effects and the pros and cons of each procedure?" They could choose from:"change," "consider to change," "not change," or "neither". The time the procedure took was calculated in seconds recorded in US and $\mathrm{C}$-arm.

The cost-effectiveness of each method was calculated with expense spent to reduce pain severity (in this study expressed in VNS reduction). The difference between the two methods was calculated with the incremental costeffective ratio (ICER), which measures the additional expense necessary for health gain of each unit as below: ${ }^{13}$

$$
\text { ICER }=\frac{\text { Average Cost }}{\text { SONO }_{\text {Average Cost }}-\text { Aluoroscopic }_{\text {Ave Effect }}}
$$

Cost is calculated with total cost of outpatient visits, and the procedure in Korean won, and effects with reduction by VNS scale. Complications before and after the procedure were also assessed.

\section{Statistics}

We performed the student's t-test to compare differences in age, BMI, and disease duration between the two groups. Normality of the sample was confirmed, with a Shapiro-Wilk test performed prior to the student's t-test. Differences in sex, capsular rupture, and left and right lesions were calculated by the chi-square test. Comparisons of VNS, SPADI, and ROM between the two groups, at each period, were performed by Shapiro-Wilk test's normality testing and by a two-way repeated measures analysis of variance ANOVA. Bonferroni's correction was completed as the post-hoc test. Procedural preferences and satisfactory levels were analyzed with the chi-square test and procedural time with the student's t-test. Statisti- 
Table 1. Characteristics of the Participants

\begin{tabular}{lccc}
\hline & A group & B group & p-value \\
\hline Number of subject & 23 & 25 & 0.756 \\
Sex (female/male) & $17(73.9 \%) / 6(26.1 \%)$ & $17(68.0 \%) / 8(32.0 \%)$ & 0.980 \\
Age (years) & $56.04 \pm 6.99$ & $56.36 \pm 8.49$ & 0.660 \\
Duration (month) & $7.65 \pm 2.33$ & $7.44 \pm 2.14$ & 0.716 \\
BMI & $22.39 \pm 2.82$ & $21.81 \pm 3.35$ & 1.000 \\
Right/Left & $12(52.2 \%) / 11(47.8 \%)$ & $10(56.0 \%) / 11(44.0 \%)$ & 0.771 \\
$\begin{array}{l}\text { Capsular distension with and } \\
\text { without rupture }\end{array}$ & $11(47.8 \%) / 12(52.2 \%)$ & & \\
\hline
\end{tabular}

Values are mean \pm standard deviation

A group: Sono-guided capsular distension group, B group: Fluoroscopically guided capsular distension group

Table 2. Comparison of the Effect on VNS by the Capsular Distension

\begin{tabular}{lccc}
\hline & $\begin{array}{c}\text { Before } \\
\text { capsular } \\
\text { distension }\end{array}$ & $\begin{array}{c}\text { 2 weeks after } \\
\text { capsular } \\
\text { distension }\end{array}$ & $\begin{array}{c}\text { 6 weeks after } \\
\text { capsular } \\
\text { distension }\end{array}$ \\
\hline A group & $7.07 \pm 0.76$ & $2.84 \pm 0.66^{*}$ & $2.41 \pm 0.64^{*}$ \\
B group & $6.92 \pm 0.61$ & $3.09 \pm 0.52^{*}$ & $2.79 \pm 0.53^{*}$ \\
\hline
\end{tabular}

Values are mean \pm standard deviation

VNS: Visual numeric scale, A group: Sono-guided capsular distension group, B group: Fluoroscopically guided capsular distension group

${ }^{*} \mathrm{p}<0.05$ : Comparison of before vs after injection, $\mathrm{p}<0.05$ : Comparison of the A group vs B group

cal analysis was processed using SAS Enterprise Guide 4.1 (4.1.0.471); the significant p-value was set below 0.05 .

\section{RESULTS}

\section{General characteristics of patients}

Group A was composed of 23 patients (6 males and 17 females) with a mean age of 56.04 years. The mean duration of disease among this group was 7.65 months. Group B was composed of 25 patients ( 8 males and 17 females) with a mean age of 56.36 and, among this group, the mean disease duration was 7.44 months. The general characteristics of patients did not differ significantly between the two groups ( $\mathrm{p}>0.05)$ (Table 1).

\section{Change in pain severity by visual numeric scale}

VNS before the procedure was $7.07 \pm 0.76 \mathrm{~cm}$ in group A and $6.92 \pm 0.61 \mathrm{~cm}$ in group B. VNS measured 2 weeks after the procedure was $2.84 \pm 0.66 \mathrm{~cm}$ and $3.09 \pm 0.52 \mathrm{~cm}$ for groups A and B respectively. Six weeks after the procedure,
Table 3. Comparison of the Effect on SPADI by the Capsular Distension

\begin{tabular}{lccc}
\hline & $\begin{array}{c}\text { Before } \\
\text { capsular } \\
\text { distension }\end{array}$ & $\begin{array}{c}\text { 2 week after } \\
\text { capsular } \\
\text { distension }\end{array}$ & $\begin{array}{c}\text { 6 weeks after } \\
\text { capsular } \\
\text { distension }\end{array}$ \\
\hline A group & $64.94 \pm 9.54$ & $31.01 \pm 9.31^{*}$ & $29.25 \pm 8.33^{*}$ \\
B group & $67.81 \pm 9.14$ & $30.02 \pm 6.55^{*}$ & $27.83 \pm 6.79^{*}$ \\
\hline
\end{tabular}

Values are mean \pm standard deviation

SPADI: Shoulder pain and Disability index, A group: Sono-guided capsular distension group, B group: Fluoroscopically guided capsular distension group ${ }^{*} \mathrm{p}<0.05$ : Comparison with the before injection

the measurements were $2.41 \pm 0.64 \mathrm{~cm}$ and $2.79 \pm 0.53 \mathrm{~cm}$ respectively, showing significant reduction in both groups. The degree of improvement and change in duration in the two groups showed no statistical differences ( $p>0.05$ ) (Table 2).

\section{Change in shoulder pain and disability index}

SPADI before the procedure was $64.94 \pm 9.54$ and $67.81 \pm 9.14$ in groups A and B respectively. SPADI, measured 2 weeks after the final procedure, was $31.01 \pm 9.31$ and $30.02 \pm 6.55$. After 6 weeks, it was $29.25 \pm 8.33$ and $27.83 \pm 6.79$ for groups A and B respectively; this shows significant reduction in both groups. The degree of improvement and change over time, for both groups, showed no statistical differences ( $p>0.05$ ) (Table 3).

Change in articular range of motion in passive movements

Passive range of motion, before and after the procedure, showed improvement for both groups and no statistical differences between the two groups ( $\mathrm{p}>0.05$ ) (Table 4). 
Change in articular range of motion in active movements

Active range of motion before and after the procedure showed improvement for both groups and did not show statistical differences between the two groups $(p>0.05)$ (Table 5).

Comparison of effectiveness, preference, time interval, cost, and side effects

There were no significant differences in effectiveness between the two groups groups, but the preference levels among group A were higher than in group $B(p<0.05)$ (Table 6). The time interval in group A was $119.04 \pm 12.17$ seconds, which was shorter than in group $B$, which was $294.08 \pm 24.30(p<0.05)$ (Table 6). Hot flush, as a side effect, was seen in 2 patients from each group, with no statistical differences between the two groups; no other side effects were reported.

The difference in VNS measured before the procedure and 6 weeks after it was $\Delta=4.58$ and $\Delta=4.14$ in groups A and $B$ respectively (Table 7 ). The total cost of the procedure for group A was 184,284 Korean won and 283,210 Korean won in group B. The ICER was calculated as 224,831 won per VNS unit. Accordingly, group A's procedure cost less and showed superior outcomes. To acquire the treatment effect of VNS 0.44 units with sono-guided capsular distension, the cost was an additional 98,926 won, compared with the fluoroscopic-assisted method.

\section{DISCUSSION}

The capsular distension method separates adherent synovium by hydropressure caused by injection of a large amount of fluid mixture. Since Salter had reported its

Table 4. Comparison of the Effect on Passive ROM by the Capsular Distension

\begin{tabular}{lcccc}
\hline Movement & Group & $\begin{array}{c}\text { Before capsular } \\
\text { distension }\end{array}$ & $\begin{array}{c}\text { 2 weeks after capsular } \\
\text { distension }\end{array}$ & $\begin{array}{c}\text { 6 weeks after capsular } \\
\text { distension }\end{array}$ \\
\hline Flexion & A & $126.74 \pm 10.18$ & $148.70 \pm 8.56^{*}$ & $152.17 \pm 10.53^{*}$ \\
& B & $123.80 \pm 9.16$ & $144.60 \pm 8.28^{*}$ & $147.00 \pm 9.12^{*}$ \\
Abduction & A & $99.57 \pm 10.54$ & $132.83 \pm 9.98^{*}$ & $134.57 \pm 10.53^{*}$ \\
& B & $100.80 \pm 13.75$ & $129.80 \pm 6.69^{*}$ & $131.40 \pm 9.52^{*}$ \\
External & A & $43.91 \pm 8.25$ & $63.91 \pm 8.25^{*}$ & $66.74 \pm 6.50^{*}$ \\
rotation & B & $45.00 \pm 9.79$ & $63.80 \pm 7.39^{*}$ & $64.60 \pm 5.39^{*}$ \\
\hline
\end{tabular}

Values are mean \pm standard deviation

ROM: Range of Motion, A group: Sono-guided capsular distension group, B group: Fluoroscopically guided capsular distension group

${ }^{*} p<0.05$ : Comparison of the before vs after the injection, $p<0.05$ : Comparison with the 2 week after the injection, $\mathrm{p}<0.05$ : Comparison of the A group vs B group

Table 5. Comparison of the Effect on Active ROM by the Capsular Distension

\begin{tabular}{lcccc}
\hline Movement & Group & $\begin{array}{c}\text { Before capsular } \\
\text { distension }\end{array}$ & $\begin{array}{c}\text { 2 weeks after capsular } \\
\text { distension }\end{array}$ & $\begin{array}{c}\text { 6 weeks after capsular } \\
\text { distension }\end{array}$ \\
\hline Flexion & $\mathrm{A}$ & $109.57 \pm 12.87$ & $139.57 \pm 8.52^{*}$ & $143.91 \pm 9.53^{*}$ \\
& $\mathrm{~B}$ & $109.40 \pm 9.61$ & $135.80 \pm 9.86^{*}$ & $139.80 \pm 10.26^{*}$ \\
Abduction & $\mathrm{A}$ & $88.70 \pm 10.89$ & $123.48 \pm 10.71^{*}$ & $127.39 \pm 8.51^{*}$ \\
& $\mathrm{~B}$ & $91.20 \pm 12.44$ & $119.62 \pm 6.29^{*}$ & $126.40 \pm 11.41^{*}$ \\
External & $\mathrm{A}$ & $36.09 \pm 9.04$ & $60.22 \pm 8.05^{*}$ & $61.74 \pm 6.50^{*}$ \\
rotation & $\mathrm{B}$ & $39.20 \pm 9.32$ & $57.40 \pm 7.23^{*}$ & $59.60 \pm 5.39^{*}$ \\
\hline
\end{tabular}

Values are mean \pm standard deviation

ROM: Range of Motion, A group: Sono-guided capsular distension group, B group: Fluoroscopically guided capsular distension group

${ }^{*} \mathrm{p}<0.05$ : Comparison of before vs after injection, $\mathrm{p}<0.05$ : Comparison with the 2 week after the injection, $\mathrm{p}<0.05$ : Comparison of the A group vs B group 
Table 6. Comparison of Effectiveness, Preference, Time Interval by the Capsular Distension

\begin{tabular}{|c|c|c|c|c|c|}
\hline \multicolumn{5}{|c|}{ Approach method } & \multirow[t]{2}{*}{ p-value } \\
\hline \multicolumn{5}{|c|}{ Effectiveness } & \\
\hline & Excellent & Good & Fair & Poor & \\
\hline A group & $3(13.0 \%)$ & $15(65.2 \%)$ & $5(21.7 \%)$ & $0(0 \%)$ & \\
\hline B group & $3(12.0 \%)$ & $15(60.0 \%)$ & $7(28.0 \%)$ & $0(0 \%)$ & 0.882 \\
\hline \multicolumn{6}{|c|}{ Preference } \\
\hline & Not change & Consider & Change & Neither & \\
\hline A group & 14 & 5 & 2 & 2 & \\
\hline B group & 4 & 5 & 10 & 6 & 0.005 \\
\hline \multicolumn{6}{|c|}{ Time intervals (sec) } \\
\hline A group & \multicolumn{4}{|c|}{$119.04 \pm 12.17$} & \\
\hline B group & \multicolumn{4}{|c|}{$294.08 \pm 24.30$} & 0.008 \\
\hline
\end{tabular}

Values are mean \pm standard deviation

A group: Sono-guided capsular distension group, B group: Fluoroscopically guided capsular distension group

effectiveness in 1970, it is widely used for its low cost, safety, and immediate effect in ROM, in patients with adhesive capsulitis. ${ }^{14}$ Rizk et al. ${ }^{6}$ explained that capsular distension shares a similar mechanism with manual reduction under anesthesia, and patients with no improvement after 3 months of conservative management should be treated.

Although controversy still exists regarding whether the primary pathology of adhesive capsulitis is inflammation or fibrosis, it is thought that its development is limited to the capsule with the synovial inner layer and synovial fluid. ${ }^{15}$ Rodeo et al. ${ }^{16}$ reported that cytokines such as Transforming Growth Factor- $\beta$ (TGF- $\beta$ ) and PlateletDerived Growth Factor (PDGF) play critical roles in the inflammatory reaction and fibrosis, and the differentiation process of fibroblasts was observed. Steroid injection was started as treatment based on the hypothesis that the main pathophysiology of capsulitis is an inflammatory response.

Neviaser ${ }^{17}$ reported mild synovial inflammation accompanied by edema, vasodilation, and the deposition of mononuclear cells in some patients with adhesive capsulitis.

As inflammation also plays an important role in the process of synovial fibrosis, anti-inflammatory treatment is necessary for symptom improvement and prevention of further fibrosis. Thus, intracapsular steroid injection in adhesive capsulitis shows efficacy by reducing inflammatory response in the pathological process. ${ }^{18}$ Additionally, if performed accurately, it produces faster pain control
Table 7. Sono-guided Capsular Distension and Fluoroscopically Guided Capsular Distension Costs

\begin{tabular}{lrr}
\hline & A group & B group \\
\hline $\begin{array}{l}\text { Room Utilization (won) } \\
\text { Procedure (won) }\end{array}$ & 60,000 & 90,840 \\
\hline $\begin{array}{l}\text { Drug (triamcinolone, lidocaine, } \\
\text { normal saline) (won) }\end{array}$ & 3,129 & 3,129 \\
Shoulder AP, LAT (won) & 11,774 \\
Technician (won) & 41,301 & 41,301 \\
Doctor's bill (won) & 68,080 & 68,080 \\
\hline Total & 184,284 & 283,210 \\
\hline
\end{tabular}

A group: Sono-guided capsular distension group, B group: Fluoroscopically guided capsular distension group

and ROM improvement with lower cost compared to conservative physiotherapy; thus, it has recently been widely used for outpatients. ${ }^{19-21}$

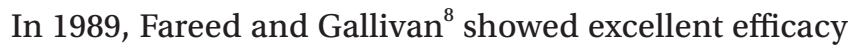
of $40 \mathrm{ml}$ steroid and saline mixture injection treatment, without individuals needing to be admitted to the hospital or needing to use arthroscope. Gam et al. ${ }^{5}$ reported that patients who received capsular distension with steroids, via a US-guided posterior approach, showed more ROM improvement and reduced use of painkillers compared with patients who received intra-articular steroid injections. The initial mechanisms of hydraulic distension are capsular rupture and pressure-lowering by capsular volume extension, then anti-inflammatory effects produced by administration of steroid, local anesthetics, and contrast media. ${ }^{6}$ In this study, additional steroid in- 
jection with capsular distension showed improvement in ROM, pain, and functional ability.

Several studies have investigated injection-targeting accuracy in blind trials. Yamakado ${ }^{22}$ reported that, when evaluated with arthrography, targeting accuracy of reaching the subacromial bursa was judged to be about $70 \%$. Partington and Broome. ${ }^{23}$ reported the targeting accuracy to be about $83 \%$ in reaching the subacromial bursa and $60 \%$ in reaching the acromioclavicular joint with blind injection on cadavers. Erroneous decisions in diagnosis and treatment efficacy evaluation can be made with inaccurate injection. The US-guided and fluoroscopic methods used in this study can reduce such errors.

Diagnostic tools for adhesive capsulitis are: arthrography, magnetic resonance imaging (MRI), arthroscopy, and musculoskeletal ultra-sonography. Arthrography displays loss of axillary folds and biceps and reduction of articular volume below $10 \mathrm{ml}$. It is usually introduced as the examination of choice, ${ }^{1,24}$ but has the disadvantage of being too invasive to be easily clinically applicable; it also has diagnostic limitations in patients whose articular volume has not decreased below $10 \mathrm{ml}$. Additionally, the risk of radiological exposure to both patients and examiner is high. Additional radiological studies may be necessary in order to rule out other rotator cuff diseases.

Holsebeeck and Introcaso. ${ }^{25}$ reported sonographic findings of adhesive capsulitis as continuous limitations of subacromial suprasinatus sliding movements in shoulder abduction and the lateral view of supraspinatus. Sonography enables diagnosis during direct monitoring of patients' movements, without risk of radiological exposure. Furthermore, sonographic measurement of the coracohumeral ligament's thickness in the girdle band is useful in diagnosing adhesive capsulitis. ${ }^{26}$

In performing sonographic capsular distension, it was difficult to access the full view of the capsule, as in fluoroscopy. To compensate for this defect, distension was confirmed during drug injection, and an additional fluoroscopic exam using contrast media was performed in order to check the shoulder capsule following drug injection.

Efficacy for the two groups showed no statistical differences but with regard to preference, 24 patients (50\%) of the total study population chose sonographic capsular distension and had higher preferences for it than fluoroscopic distension, which was chosen by only 6 patients

\section{(12.5\%).}

The main reason patients preferred sonographic distension was lack of radiological exposure. X-rays during fluoroscopic distension mean a type of ionizing radiation and have potent biological effects. Even a small amount can cause molecular damage and be carcinogenic in the future. ${ }^{27}$ The International Commission of Radiological Protection estimates the annual maximal permissible dose on several organs and recommends that an examiner or patient not be exposed to more than $10 \%$ of the permissible dose. ${ }^{28}$ The usual incidental skin dose during a one-minute fluoroscopic interventional procedure is 1-10 R (Roentgen) with a $2 \mathrm{R}$ per minute ray, equal to a radiological exposure of 130 times a chest radiograph, as an incidental skin dose of a typical chest X-ray is $15 \mathrm{mR}$ (milliroentgen). ${ }^{25}$ Thus, a procedure without risk of radiological exposure is preferred.

A total of 8 patients, 2 in group A and 6 in group B, refused both procedures because of pain during involved in them. As pain can reduce patients' compliance to procedures, further studies ought to be performed in this field.

The limitations of this study were the small number of patients and the short (6-week) follow-up period. Enrollment criteria included patients of 1 and 2 grade adhesive capsulitis, with symptom duration lasting between 3 months and 2 years, but grade 3 patients could have been included for period overlap. Additionally, the cost was calculated for four different hospitals, for reliable results in evaluating cost-effectiveness. However, the part of the cost not covered by insurance differed among hospitals and was a limitation in analysis of cost-effectiveness. Even though individuals were examined by an expert with 5 years of experience in musculoskeletal ultrasonography and C-arm, the procedure time differences according to inter-examiners' performance abilities remains a limitation.

A larger number of subjects, with a longer follow-up period comparing multiple methods of capsular distension, are needed in order to fully assess efficacy and costeffectiveness in the future.

\section{CONCLUSION}

In the comparison of sono-guided capsular distension with fluoroscopically guided capsular distension in adhesive capsulitis, results showed no statistical differences in 
ROM improvement and pain control. However, the sonoguided capsular distension method is preferred among patients due to lack of radiological exposure, lower cost, and faster procedure time.

\section{REFRERENCES}

1. Binder AI, Bulgen DY, Hazleman BL, Roberts S. Frozen shoulder: a long-term prospective study. Ann Rheum Dis 1984; 43: 361-364

2. Neviaser TJ. Arthroscopy of the shoulder. Orthop Clin North Am 1987; 18: 361-372

3. Andersen NH, Søjbjerg JO, Johannsen HV, Sneppen O. Frozen shoulder: arthroscopy and manipulation under general anesthesia and early passive motion. J Shoulder Elbow Surg 1998; 7: 218-222

4. Murnaghan JP. Adhesive capsulitis of the shoulder: current concepts and treatment. Orthopedics 1988; 11: 153-158

5. Gam AN, Schydlowsky P, Rossel I, Remvig L, Jensen EM. Treatment of "frozen shoulder" with distension and glucorticoid compared with glucorticoid alone. A randomised controlled trial. Scand J Rheumatol 1998; 27: 425-430

6. Rizk TE, Gavant ML, Pinals RS. Treatment of adhesive capsulitis (frozen shoulder) with arthrographic capsular distension and rupture. Arch Phys Med Rehabil 1994; 75: 803-807

7. Vad VB, Sakalkale D, Warren RF. The role of capsular distention in adhesi ve capsulitis. Arch Phys Med Rehabil 2003; 84: 1290-1292

8. Fareed DO, Gallivan WR Jr. Office management of frozen shoulder syndrome. Treatment with hydraulic distension under local anesthesia. Clin Orthop Relat Res 1989; 242: 177-183

9. Tveita EK, Tariq R, Sesseng S, Juel NG, Bautz-Holter E. Hydrodilatation, corticosteroids and adhesive capsulitis: a randomized controlled trial. BMC Musculoskelet Disord 2008; 9: 53

10. Arslan S, Celiker R. Comparison of the efficacy of local corticosteroid injection and physical therapy for the treatment of adhesive capsulitis. Rheumatol Int 2001; 21: $20-23$

11. Calis M, Demir H, Ulker S, Kirnap M, Duygulu F, Calis HT. Is intraarticular sodium hyaluronate injection an alternative treatment in patients with adhesive capsu- litis? Rheumatol Int 2006; 26: 536-540

12. Roach KE, Budiman-Mak E, Songsiridej N, Lertratanakul Y. Development of a shoulder pain and disability index. Arthritis Care Res 1991; 4: 143-149

13. Zaghi S, Heine N, Fregni F. Brain stimulation for the treatment of pain: a review of costs, clinical effects, and mechanisms of treatment for three different central neuromodulatory approaches. J Pain Manag 2009; 2: 339-352

14. Fareed DO, Gallivan WR Jr. Office management of frozen shoulder syndrome. Treatment with hydraulic distension under local anesthesia. Clin Orthop Relat Res 1989; 242: 177-183

15. Mclaughlin HL. On the frozen shoulder. Bull Hosp Joint Dis 1951; 12: 383-393

16. Rodeo SA, Hannafin JA, Tom J, Warren RF, Wickiewicz TL. Immunolocalization of cytokines and their receptors in adhesive capsulitis of the shoulder. J Orthop Res 1997; 15: 427-436

17. Neviaser JS. Adhesive capsulitis of the shoulder : a study ofthe pathologic findings in periarthritis of the shoulder. J Bone Joint Surg 1945; 27: 211-222

18. de Jong BA, Dahmen R, Hogemwg JA, Marti RK. Intraarticular triamcinolone acetonide injection in patients with capsulitis of the shoulder: a comparative study of two dose regimens. Clin Rehabil 1998; 12: 211-215

19. Bulgen DY, Binder AI, Hazleman BL, Dutton J, Roberts S. Frozen shoulder: prospective clinical study with an evaluation of three treatment regimens. Ann Rheum Dis 1984; 43: 353-360

20. Dacre JE, Beeney N, Scott DL. Injections and physiotherapy for the painful stiff shoulder. Ann Rheum Dis 1989; 48: 322-325

21. Rizk TE, Pinals RS, Talaiver AS. Corticosteroid injections in adhesive capsulitis: investigation of their value and site. Arch Phys Med Rehabil 1991; 72: 20-22

22. Yamakado K. The targeting accuracy of subacromial injection to the shoulder: an arthrographic evaluation. Arthroscopy 2002; 18: 887-891

23. Partington PF, Broome GH. Diagnostic injection around the shoulder: hit and miss? A cadaveric study of injection accuracy. J Shoulder Elbow Surg 1998; 7: 147-150

24. Connell D, Padmanabhan R, Buchbinder R. Adhesive capsulitis: role of MR imaging in differential diagnosis. Eur Radiol 2002; 12: 2100-2106 
25. Van Holsbeeck MT, Introcaso JH. Sonography of the shoulder. In: Ptasznik R, Editor. Musculoskeletal ultrasound, 2nd ed, St Louis, Mosby Inc, 2001, 504-505

26. Homsi C, Bordalo-Rodrigues M, da Silva JJ, Stump $\mathrm{XM}$. Ultrasound in adhesive capsulitis of the shoulder: is assessment of the coracohumeral ligament a valu- able diagnostic tool? Skeletal Radiol 2006; 35: 673-678 27. Berlin L. Radiation-induced skin injuries and fluoroscopy. AJR Am J Roentgenol 2001; 177: 21-25

28. Fishman SM, Smith H, Meleger A, Seibert JA. Radiation safety in pain medicine. Reg Anesth Pain Med 2002; 27: 296-305 